\title{
Modeling Travel Time Reliability of Road Network Considering Connected Vehicle Guidance Characteristics Indexes
}

\author{
Jiangfeng Wang, Chao Wang, Jiarun Lv, Zhiqi Zhang, and Cuicui Li \\ MOE Key Laboratory for Transportation Complex Systems Theory and Technology, Beijing Jiaotong University, \\ Beijing 100044, China \\ Correspondence should be addressed to Jiangfeng Wang; wangjiangfeng@bjtu.edu.cn
}

Received 10 January 2017; Revised 18 February 2017; Accepted 14 March 2017; Published 29 March 2017

Academic Editor: Xiaobo Qu

Copyright (C) 2017 Jiangfeng Wang et al. This is an open access article distributed under the Creative Commons Attribution License, which permits unrestricted use, distribution, and reproduction in any medium, provided the original work is properly cited.

\begin{abstract}
Travel time reliability (TTR) is one of the important indexes for effectively evaluating the performance of road network, and TTR can effectively be improved using the real-time traffic guidance information. Compared with traditional traffic guidance, connected vehicle $(\mathrm{CV})$ guidance can provide travelers with more timely and accurate travel information, which can further improve the travel efficiency of road network. Five CV characteristics indexes are selected as explanatory variables including the Congestion Level (CL), Penetration Rate (PR), Compliance Rate (CR), release Delay Time (DT), and Following Rate (FR). Based on the five explanatory variables, a TTR model is proposed using the multilogistic regression method, and the prediction accuracy and the impact of characteristics indexes on TTR are analyzed using a CV guidance scenario. The simulation results indicate that $80 \%$ of the RMSE is concentrated within the interval of 0 to 0.0412 . The correlation analysis of characteristics indexes shows that the influence of CL, PR, CR, and DT on the TTR is significant. PR and CR have a positive effect on TTR, and the average improvement rate is about $77.03 \%$ and $73.20 \%$ with the increase of PR and CR, respectively, while CL and DT have a negative effect on TTR, and TTR decreases by $31.21 \%$ with the increase of DT from 0 to $180 \mathrm{~s}$.
\end{abstract}

\section{Introduction}

Travel time reliability (TTR) is an important concern issue for traveler in daily travel and is influenced by various factors such as traffic accidents and weather and flow states. Traffic guidance information can help travelers make better travel plans and improve their travel efficiency and TTR. Compared to the traditional variable message signs, connected vehicle $(\mathrm{CV})$ guidance can in real time transmit traffic information through vehicle-to-vehicle $(\mathrm{V} 2 \mathrm{~V})$ communication and vehicle-to-infrastructure (V2I) communication, which make traffic guidance information released in a more timely manner, and can effectively improve the effect of guidance [1-3].

Previous studies on TTR and CV guidance have mainly focused on analyzing the impact of traditional factors such as weather [4-8] and the impact of CV guidance on the travel time, fuel consumption, and average delay [9-12]. Few studies were conducted on the impact of $\mathrm{CV}$ guidance on TTR considering the CV characteristics indexes. Based on the current research status, a TTR model in a CV environment was presented using the multilogistic regression method.
In order to validate the effectiveness of the proposed TTR model, a CV guidance simulation scenario was established to analyze the prediction accuracy of the proposed model and to explore the interaction between $\mathrm{CV}$ characteristics indexes and TTR in a CV environment.

\section{Literatures Review}

Many scholars have conducted the numerous basic studies on TTR of road networks, and several definitions of TTR were proposed. Asakura and Kashiwadani [4] first proposed the concept of TTR considering the travel demands of road networks. Iida [13] also presented a definition for TTR using a probability theory, which is defined as the probability of traveler successfully reaching their destination within a specified time. Liu et al. [14] studied the TTR of stochastic road networks using an approximation algorithm based on the user equilibrium distribution model. Based on the user equilibrium and punishing user equilibrium models, Wang et al. [15] proposed a biobjective user equilibrium model for 
analyzing the TTR. In addition, Pei and Gai [16] analyzed the TTR of a road network using the Monte Carlo simulation method. Based on the previous studies, several scholars proposed some evaluation indexes to further analyze the TTR [17-20].

Aimed at the impact of abnormal conditions on TTR of road networks, such as traffic demands, adverse weather, and road feature, many studies were conducted [21-23]. Asakura [24] analyzed the impact of variable traffic demand on TTR. Tavassoli Hojati et al. [5] modeled and analyzed TTR under the influence of Traffic Accident. As one of the important influence factors, many studies were focused on the impact of adverse weather on TTR. Leng [6] studied the TTR for urban road networks under the conditions of snow and ice and analyzed the traffic characteristics under such conditions. $\mathrm{Wu}$ et al. and Wang et al. [7, 8] explored the impact of fog and rainy conditions on freeway networks using the proposed evaluation indexes. The above results indicate that the traffic demand, poor weather, and Traffic Accidents all have a significant impact on TTR. However, less research has been conducted on the impact of $\mathrm{CV}$ guidance characteristics indexes on TTR.

Comparing to traditional traffic guidance, $\mathrm{CV}$ guidance can in real time share the traffic information through V2V and V2I communication, which were effective methods to improve the guidance effect of road networks. Lee and Park [9] analyzed the performance of CV guidance under accident conditions using simulation, and the results indicated that Penetration Rate of the CV equipment has a significant impact on the guidance effect. Yang et al. [25] found that the total travel time, average travel time, and average delay of a vehicle in a CV environment were significantly lower than under a traditional traffic environment. Noori and Valkama [26] also found that a dynamic route guidance strategy based on CV technology can reduce the travel time by $52 \%$ and the fuel consumption by $48 \%$. Nie [27] studied the impact of V2V communication on traffic flow, and the results showed that $\mathrm{V} 2 \mathrm{~V}$ communication can reduce the average travel time. In addition, Paikari et al. [28] analyzed the impact of Following Rate, and the results indicated that $\mathrm{V} 2 \mathrm{~V}$ communication not only can reduce the travel time of vehicles with CV equipment but can also reduce the travel time of vehicles without $\mathrm{CV}$ equipment.

Generally, CV guidance characteristics, such as Penetration Rate and Following Rate, have a significant effect on TTR of road networks in a CV environment $[29,30]$. It is very essential to establish comprehensive characteristics indexes for $\mathrm{CV}$ guidance and to analyze the impact of characteristics indexes on TTR of road networks.

\section{Methodology}

3.1. Definition of TTR. By referring to the available research, TTR is defined as the probability of a traveler successfully completing a trip within a specified time in this study, as shown in Figure 1.

According to the definition, the TTR formula is

$$
R(t)=\operatorname{Pr}\left\{t_{i} \leq \Phi\right\},
$$

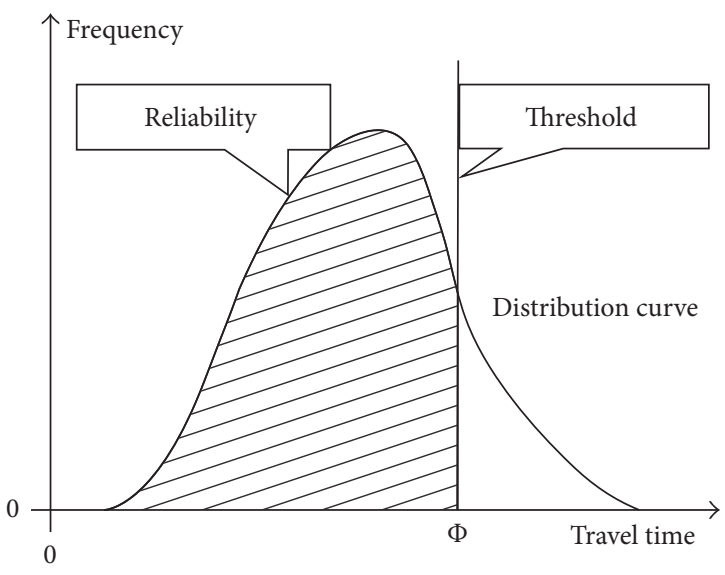

FIGURE 1: Definition of TTR.

where $\Phi$ is the travel time threshold (s), which is related to the service level. The travel time threshold $\Phi$ is calculated by

$$
\Phi=\bar{t}_{a} \times l_{a},
$$

where $\bar{t}_{a}$ is the travel time of unit distance $a(\mathrm{~s})$, which can be found in the HCM. $l_{a}$ is the length of route $a(\mathrm{~km})$.

3.2. CV Characteristics Indexes. Based on the above-mentioned references, the $\mathrm{CV}$ guidance characteristics indexes will affect the TTR of road networks in a CV environment. To explore the impact of characteristics indexes, the characteristics indexes system is established, as shown in Figure 2.

In this study, the Compliance Rate (CR), Following Rate (FR), Penetration Rate (PR), release Delay Time (DT), and Congestion Level (CL) are selected as the characteristics indexes to analyze the impact of these characteristics indexes on TTR. CR is the ratio of vehicles with CV ability to comply with route adjustments to all vehicles with $\mathrm{CV}$ ability. FR refers to the ratio of vehicles without $\mathrm{CV}$ ability to adjust the route because of the influence of the leading vehicle changing the route to all vehicles without $\mathrm{CV}$ ability. PR refers to the proportion of vehicles with $\mathrm{CV}$ ability to all vehicles. DT refers to the interval from the generation of traffic information to the reception of traffic information by the vehicle. CL is an important index for evaluating the flow operation states of the road network, and the duration of congestion caused by an accident vehicle is used as an alternative variable in this paper.

3.3. TTR Model. A road network is composed of several paths, and a path selection model is established to design the TTR model for road networks. A multilogistic regression method is used to establish path selection model.

Supposing that there are $k$ paths between O-D pairs $R S$ in the $\mathrm{CV}$ guidance environment and the five $\mathrm{CV}$ guidance characteristics indexes are selected as the explanatory variables of the multilogistic model, the path $m$ is the reference route, and 


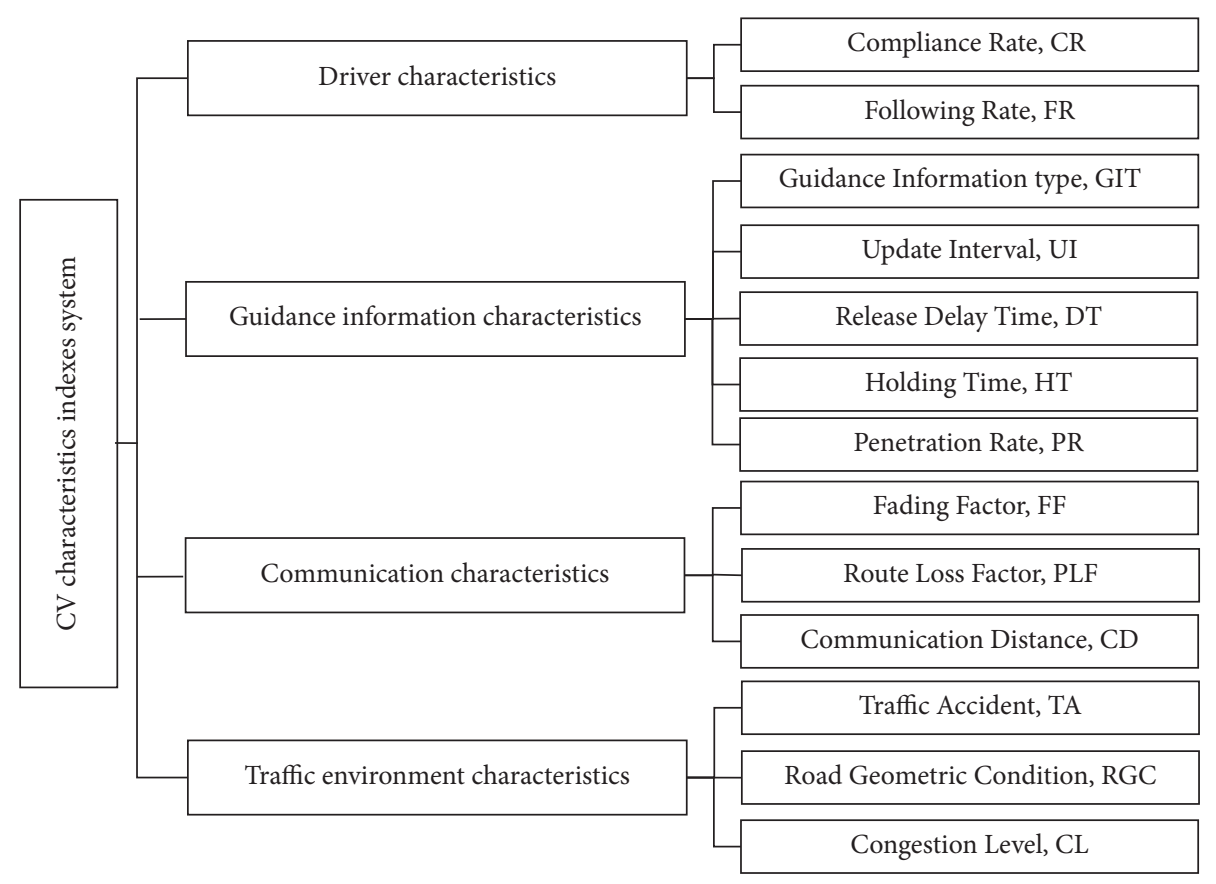

FIGURE 2: CV characteristics indexes system.

the utility function of the path $i$ relative to the reference path $m$ is

$$
U_{i}^{R S}=\ln \left(\frac{p_{i}^{R S}}{p_{m}^{R S}}\right)=\beta_{i, 0}^{R S}+\sum_{j=1}^{5} \beta_{i, j}^{R S} \cdot x_{j}
$$

where $U_{i}^{R S}$ is the utility of selecting the path $i$ relative to the path $m, i \neq m, 1 \leqslant i \leqslant k ; p_{i}^{R S}$ and $p_{m}^{R S}$ are the probability of selecting the path $i$ and path $m$, respectively; $\beta_{i, 0}^{R S}$ is a constant of the utility function for selecting the path $i ; x_{j}$ is the $\mathrm{CV}$ characteristic index $j$; and $\beta_{i, j}^{R S}$ is the coefficient of $x_{j}$ of the path $i$.

Letting $y=\sum_{i=1}^{k-1} \exp \left(U_{i}^{R S}\right)=\sum_{i=1}^{k-1}\left(p_{i}^{R S} / p_{m}^{R S}\right)$, the selection probability of path $i$ between $\mathrm{O}-\mathrm{D}$ pairs $R S$ is

$$
p_{i}^{R S}=\frac{e^{U_{i}^{R S}}}{1+y}
$$

The TTR of O-D pairs RS is obtained based on the weighted method, which is expressed as

$$
R^{R S}=\sum_{i} R_{i}^{R S} \cdot p_{i}^{R S}
$$

where $R^{R S}$ is the TTR of O-D pairs $R S$ and $R_{i}^{R S}$ is the TTR of the path $i$ between O-D pairs $R S$.

The calculation of the TTR of road network is based on the TTR of O-D pairs, and the weighted average of the TTR of all OD pairs in the road network is selected as the TTR of the network. The TTR model of road network is established in a $\mathrm{CV}$ guidance environment as follows:

$$
\begin{aligned}
R_{\mathrm{CV}}= & \sum_{R} \sum_{S} \sum_{i}\left(R_{i}^{R S} \times p_{i}^{R S}\right) \times\left(\frac{q^{R S}}{\sum_{R} \sum_{S} q^{R S}}\right) \\
= & \sum_{R} \sum_{S} \sum_{i}\left(F_{i}^{R S}\left(\Phi_{i}^{R S}\right) \times \frac{e^{U_{i}^{R S}}}{1+y}\right) \\
& \times\left(\frac{q^{R S}}{\sum_{R} \sum_{S} q^{R S}}\right),
\end{aligned}
$$

where $R_{\mathrm{CV}}$ is the TTR of the road network; $q^{R S}$ is the volume of O-D pairs $R S, \mathrm{pcu} / \mathrm{h} ; F_{i}^{R S}(\cdot)$ is the travel time distribution function of the path $i$ between the O-D pairs $R S$; and $\Phi_{i}^{R S}$ is the travel time threshold of the path $i$ between the O-D pairs $R S$.

\section{Simulation Scenario}

4.1. Experimental Scene. To evaluate the TTR model, a simple road network with three routes is selected to establish the simulation scenario, as shown in Figure 3. In the initialization stage, vehicles with connected vehicle ability (CVs) and vehicles without connected vehicle ability (non-CVs) are arranged in the road network. Car-Agent is used to control the vehicles' traffic behaviors, such as car following and lane changing by the agents programing using the EstiNet tool. The Roadside Unit (RSU) at an intersection is used to collect the volume entering the intersection and transmit the volume to the Central-Roadside Unit (C-RSU) in real 
TABLE 1: Parameter settings.

\begin{tabular}{lccccc}
\hline Route number & Route node & Passing intersection & Length & Number of lanes & Lane width \\
\hline 1 & A-B-C-M-F-G-J & B, C, F, G & $15.74 \mathrm{~km}$ & 2 & $3.5 \mathrm{~m}$ \\
2 & A-B-C-D-E-F-G-J & B, C, F, G & $15.94 \mathrm{~km}$ & 2 & $3.5 \mathrm{~m}$ \\
3 & A-B-H-I-G-J & B, G & $16.14 \mathrm{~km}$ & 2 & $3.5 \mathrm{~m}$ \\
\hline
\end{tabular}

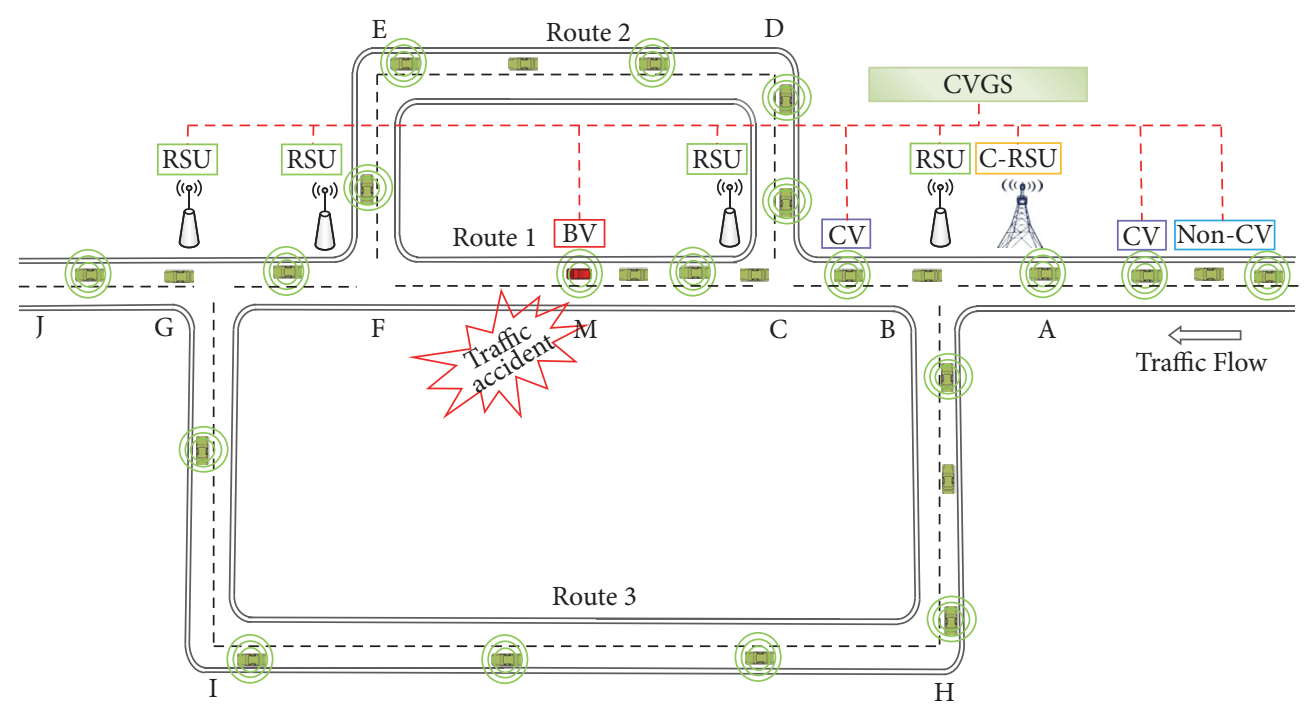

FIGURE 3: Road network structure.

time. The C-RSU is responsible for receiving the volume from the downstream intersections, which calculates the shortest route using the Bureau of Public Roads (BPR) impedance function. To generate the traffic congestion, a broken vehicle (BV) is set on Route 1. During the simulation stage, all vehicles, including $\mathrm{CV}$ and non-CV, choose the shortest route to travel depending on the real-time traffic information obtained through V2V and V2I communication. When the simulation starts, the BV sends the accident information to the C-RSU. Meanwhile, the C-RSU broadcasts the guidance information to all vehicles. $\mathrm{CV}$ s will choose the shortest route to travel according to the $\mathrm{CV}$ guidance information, and non-CVs will choose their routes to travel based on their own reasonable judgment. For example, these non-CVs may change their initial route by following the leading vehicles depending on the FR.

The basic parameters of the three routes are shown in Table 1.

Using the proposed CV guidance scenario, a guidance strategy is designed. The flow chart of this guidance strategy is shown in Figure 4.

When simulation starts, all vehicles including non-CVs and CVs travel according to the respective shortest routes. Once a Traffic Accident occurs in the shortest Route 1, the C-RSU will recalculate the shortest route using the BPR impedance function and broadcasts the guidance information to all vehicles. Non-CVs and CVs will replan their routes to travel based on the values of characteristics indexes, such as $\mathrm{CR}$ and FR.
TABLE 2: CV characteristics indexes and parameters settings.

\begin{tabular}{lc}
\hline Parameter & Value \\
\hline Simulation time (s) & 1800 \\
Maximum speed (m/s) & 18 \\
Acceleration(m/s2) & $-4 \sim 1$ \\
CL (s) & $300 / 500$ \\
PR (\%) & $0 / 25 / 50 / 75 / 100$ \\
CR (\%) & $25 / 50 / 75 / 100$ \\
FR (\%) & $0 / 10 / 20 / 30$ \\
DT (s) & $0 / 120 / 180$ \\
Number of test vehicles & 100 \\
Antenna height (m) & 1.5 \\
Average height of buildings (m) & 10 \\
Average spacing of buildings (m) & 80 \\
Communication protocol & $802.11 \mathrm{p}$ \\
Signal attenuation (dbm) & 5 \\
Frequency (GHz) & 10 \\
Communication distance (m) & 1000 \\
Signal interference range (m) & 550 \\
\hline
\end{tabular}

4.2. Experimental Scheme. CL, PR, CR, FR, and DT are selected as the influence factors in the simulation experiment. The travel time for each route could be obtained through the simulation. The CV characteristics and other parameter settings are shown in Table 2. 


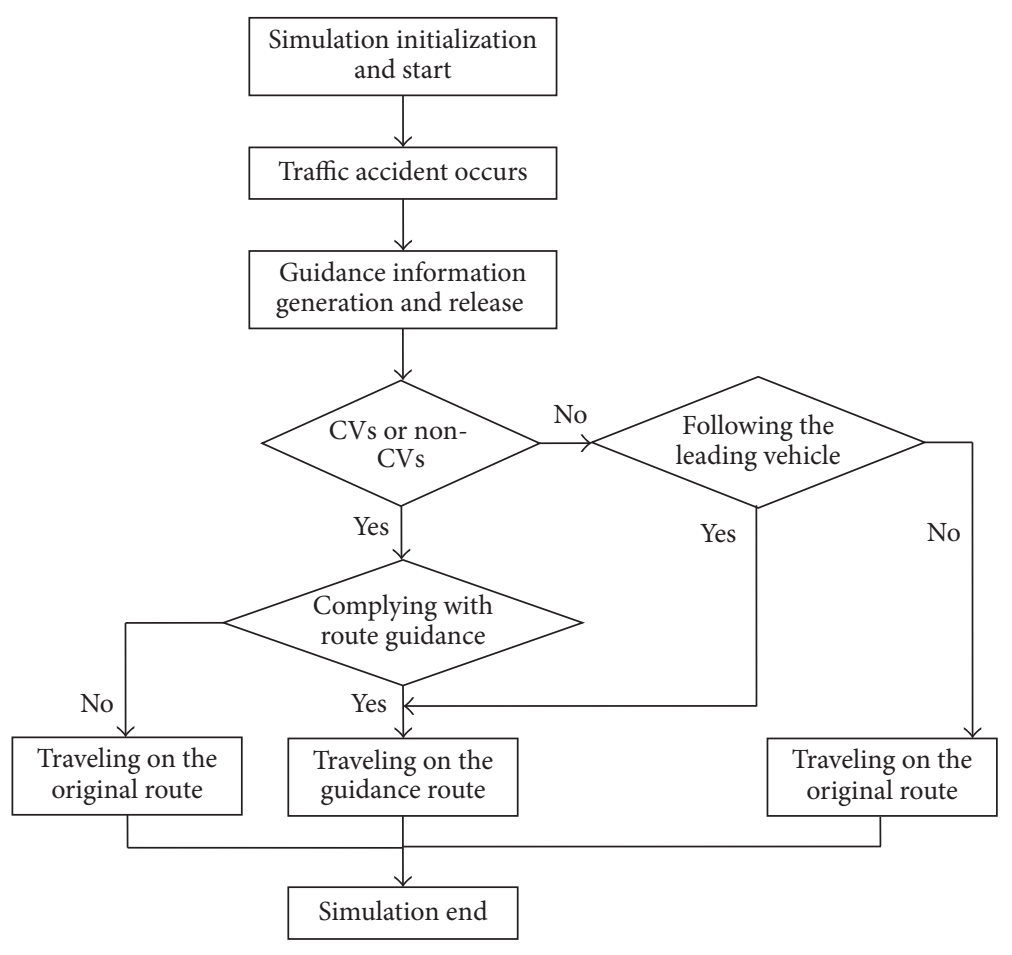

FIgURE 4: Flow chart of traffic guidance strategy.

To obtain different experimental scenarios, different values for the five CV characteristics indexes are applied. For a $\mathrm{PR}$ of zero, there are 24 scenarios $(2 \mathrm{CL} \times 1 \mathrm{PR} \times 4 \mathrm{CR} \times 1 \mathrm{FR} \times$ $3 \mathrm{DT}=24)$ used. For the different value of PR, there are 288 scenes $(2 \mathrm{CL} \times 3 \mathrm{PR} \times 4 \mathrm{CR} \times 4 \mathrm{FR} \times 3 \mathrm{DT}=288)$. A simulation is carried out for each experimental scenario, and the model is calibrated using the data from 200 of the experimental scenarios. The remaining 112 experimental scenarios are used for route prediction.

\section{Results Analysis}

5.1. Parameter Calibration. Before calibrating the model, we first verified the effectiveness of the guidance strategy. The traffic conditions for $\mathrm{CL}=500 \mathrm{~s}, \mathrm{PR}=100 \%, \mathrm{CR}=100 \%$, $\mathrm{FR}=0$, and DT $=120 \mathrm{~s}$ are selected to establish the CV guidance scenario. The proportion of volumes along each route is shown in Figure 5.

It is shown in Figure 5 that vehicles initially travel on the shortest Route 1. Traffic jam occurs in Route 1 to cause the increasing of the impedance of Route 1 when a broken vehicle (BV) is set on Route 1. The jam information is in real time transmitted to the upstream vehicles via V2I and V2V. C-RSU will replan the routes by comparison of three routes' impedance according to the guidance information. Thus vehicles will select Route 2 and Route 3 to travel. The distribution of volume in Route 2 and Route 3 gradually tends to balance in $\mathrm{CV}$ guidance environment according to the impedance of two routes.

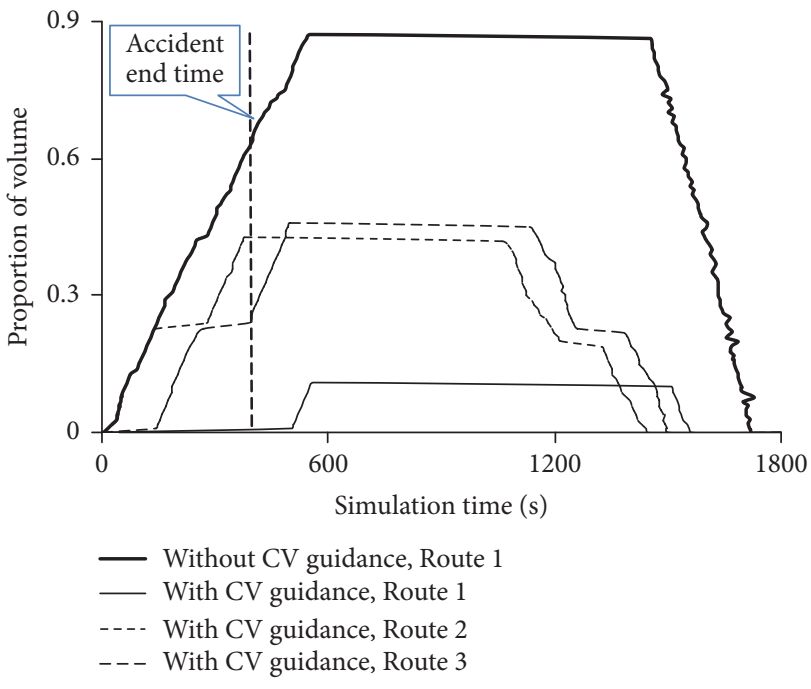

FIgURE 5: Traffic conditions of the road network.

Using the calibration samples and considering Route 3 as the reference choice route, the calibrated results are as follows:

$$
\begin{aligned}
U_{1}^{R S}= & \ln \left(\frac{p_{1}^{R S}}{p_{3}^{R S}}\right) \\
= & 7.600-0.002 \mathrm{CL}-3.142 \mathrm{PR}-3.491 \mathrm{CR} \\
& -3.739 \mathrm{FR}+0.003 \mathrm{DT},
\end{aligned}
$$




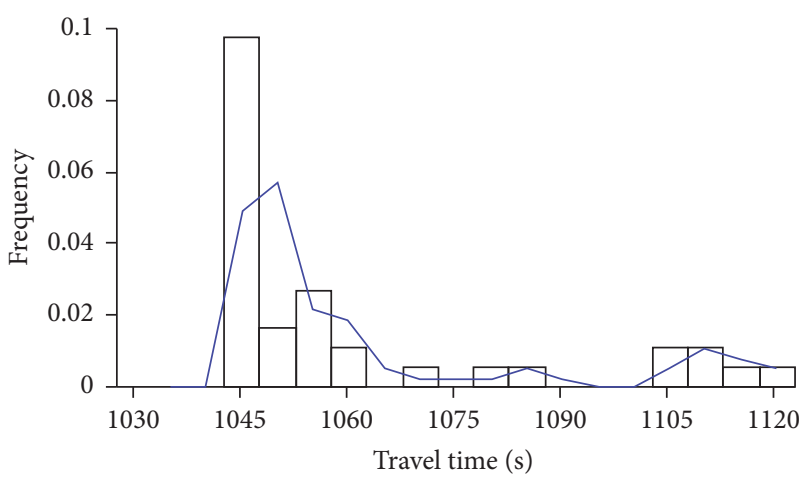

(a) Route 2

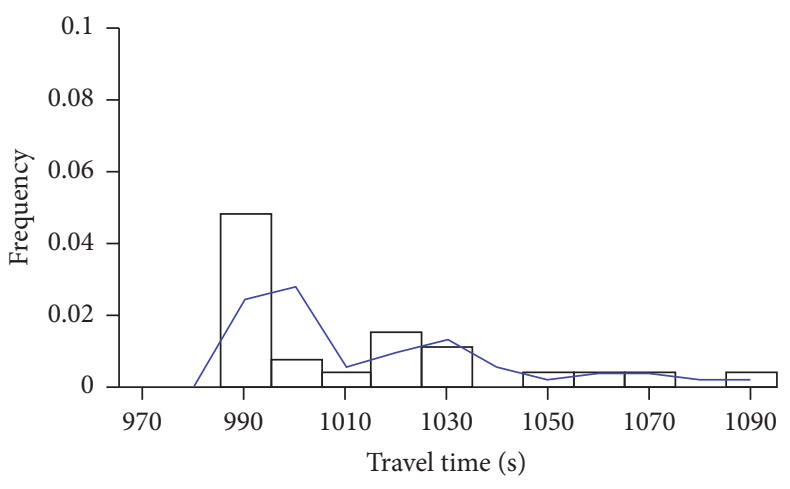

(b) Route 3

FIGURE 6: The travel time distribution of vehicles.

$$
\begin{aligned}
U_{2}^{R S}= & \ln \left(\frac{p_{2}^{R S}}{p_{3}^{R S}}\right) \\
= & 4.089-0.002 \mathrm{CL}-1.534 \mathrm{PR}-1.483 \mathrm{CR} \\
& -1.860 \mathrm{FR}+0.001 \mathrm{DT} .
\end{aligned}
$$

According to the route selection model, the selection probability of each route can be obtained as follows:

$$
\begin{aligned}
& p_{1}^{R S}=\frac{e^{U_{1}^{R S}}}{1+e^{U_{1}^{R S}}+e^{U_{2}^{R S}}}, \\
& p_{2}^{R S}=\frac{e^{U_{2}^{R S}}}{1+e^{U_{1}^{R S}}+e^{U_{2}^{R S}}}, \\
& p_{3}^{R S}=\frac{1}{1+e^{U_{1}^{R S}}+e^{U_{2}^{R S}}},
\end{aligned}
$$

where $p_{1}^{R S}, p_{2}^{R S}$ and $p_{3}^{R S}$ are the selection probability of Route 1 , Route 2 , and Route 3 in the simulation, respectively.

Based on the calibrated route selection model, the selection probability of each route is obtained, and, according to the experiment data, the TTR of road network can be determined:

$$
R_{\mathrm{CV}}=\sum_{i=1}^{3} R_{i}^{R S} \times p_{i}^{R S},
$$

where $R_{\mathrm{CV}}$ is the prediction value of TTR of road network and $R_{i}^{R S}$ is the TTR of $i$ th route, $i=1,2,3$.

5.2. Model Prediction Accuracy. To calculate the TTR of road network, the TTR of each route is first determined. Using (1), the TTR of each route can be obtained based on the travel time distribution. Therefore, the travel time distribution of vehicles in each route is analyzed, as follows in Figure 6.

Figures 6(a) and 6(b) showed the travel time distribution of the vehicles running along Route 2 and Route 3 during the simulation, respectively. The TTR for each route can be obtained according to (1) and (2), and the values of TTR of

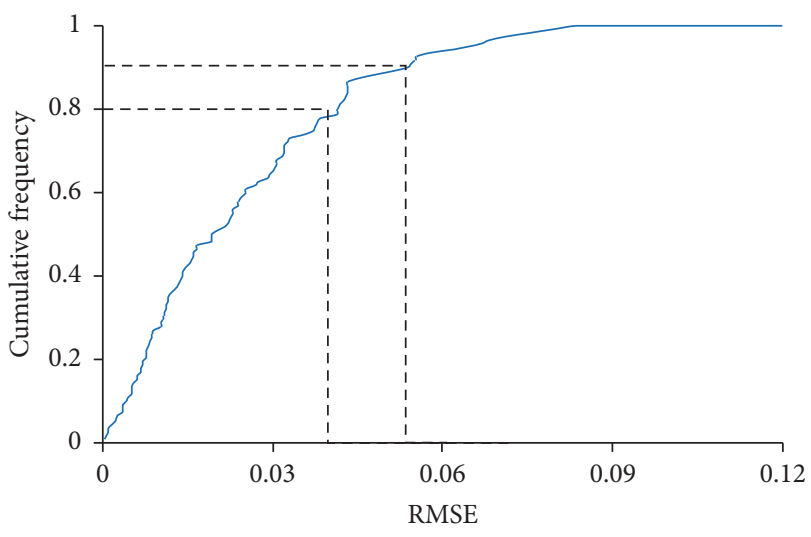

FIGURE 7: Cumulative probability distribution curve of RMSE.

road network can be obtained according to $(8) \sim(9)$. Thus the prediction accuracy of TTR model can be determined.

The cumulative probability distribution curve of the root mean square error (RMSE) is shown in Figure 7.

The results indicate that $80 \%$ of the RMSE is in the range of 0 to 0.0412 . It can be concluded that the proposed TTR model has a good prediction accuracy, which can reflect the TTR for a road network in a CV guidance environment.

5.3. Sensitivity Analysis of TTR. The 312 sets of experimental data are applied to conduct a correlation analysis, and the results are shown in Table 3 .

As can be seen from Table 3, CL, PR, CR, and DT can be considered to have a significant impact on TTR at a 0.01 significance level. To clarify the impact of these four factors on TTR, a sensitivity analysis is conducted, as shown in Figure 8 .

Figure 8(a) indicates that higher CL means lower TTR, which is consistent with common sense. When the Congestion Level is severe, TTR will decrease even if CV guidance is applied. Figure 8(b) showed that TTR increases with an increase in PR. The reason is that more vehicles can receive guidance information, and thus more vehicles will choose an alternative route to travel. When PR is increased from 0 
TABLE 3: Results of correlation analysis.

\begin{tabular}{lccccc}
\hline Factor & $\begin{array}{c}\text { Degrees of freedom } \\
\text { intergroups }\end{array}$ & $\begin{array}{c}\text { Degrees of freedom } \\
\text { intragroup }\end{array}$ & $\begin{array}{c}\text { Mean square } \\
\text { intergroups }\end{array}$ & $\begin{array}{c}\text { Mean square } \\
\text { intragroup }\end{array}$ & $\begin{array}{c}\text { Significance } \\
\text { level }\end{array}$ \\
\hline CL & 1 & 310 & 4.320 & 0.027 & 157.407 \\
PR & 3 & 308 & 0.669 & 0.000 & 19.029 \\
CR & 3 & 308 & 1.238 & 0.030 & 41.846 \\
FR & 3 & 308 & 0.117 & 0.000 & 0.000 \\
DT & 2 & 309 & 0.449 & 0.039 & 2.879 \\
\hline
\end{tabular}

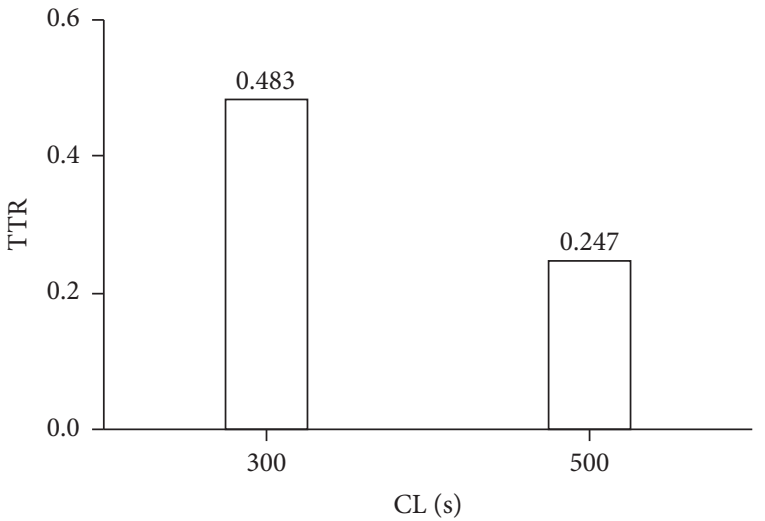

(a)

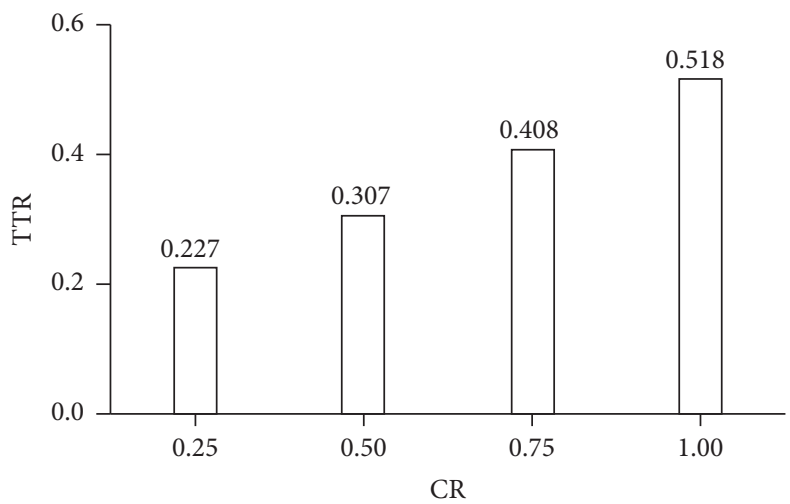

(c)

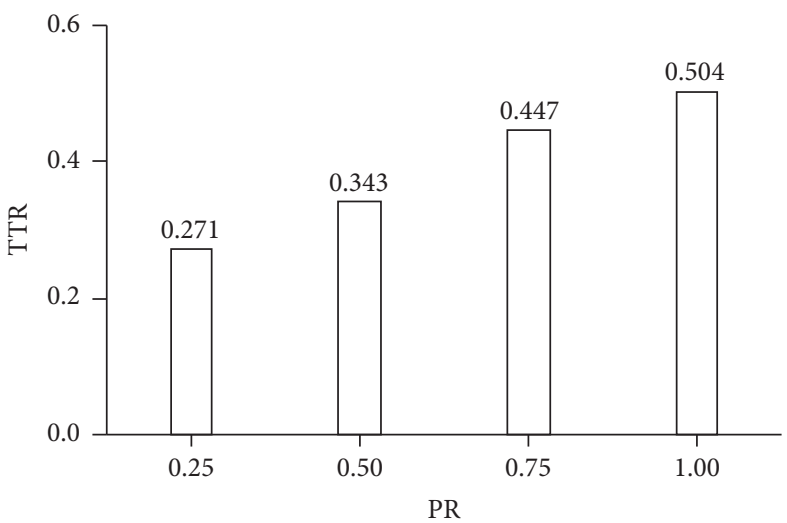

(b)

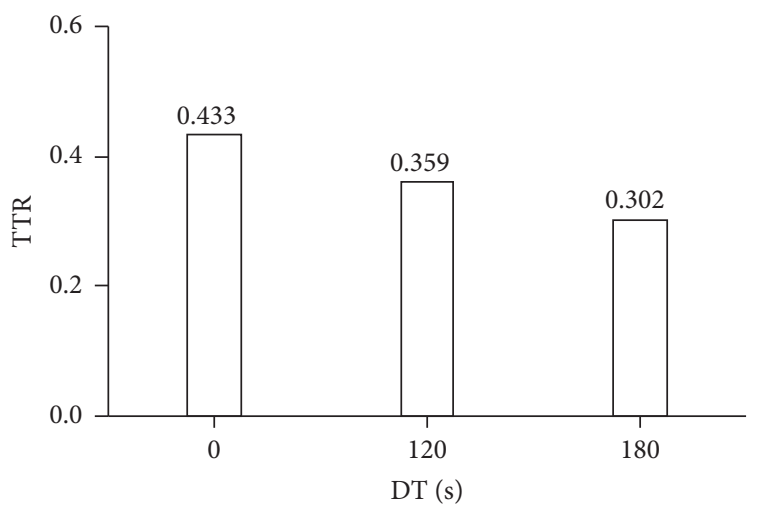

(d)

FIGURE 8: The impact of these four characteristics indexes on TTR.

to $100 \%$, TTR can be improved by $20.03 \%$ to $134.04 \%$ using CV guidance information. It can be seen from Figure 8(c) that the effect of CR on TTR is similar to that of PR. TTR gradually increases with an increase in $C R$, and the improvement trend is slower and slower. The reason for this is that an increase in CR also means more vehicles will accept the guidance information, which makes more vehicles select the lower impedance route to travel. When CR increases from $25 \%$ to $100 \%$, TTR improves from $11.08 \%$ to $135.31 \%$. It can be seen from Figure $8(\mathrm{~d})$ that TTR gradually decreases as DT increases. The reason is that the greater the delay receiving the guidance information the fewer the vehicles to detour in time, which affects the TTR.

\section{Conclusions}

In this study, a travel time reliability model in a $\mathrm{CV}$ environment is developed, and the prediction accuracy of the proposed model and its correlation with five selected characteristics indexes are analyzed. The results indicate that the prediction accuracy of the model is good, and some influence rules are summarized. (1) Among the five characteristics indexes, the influence of CL, PR, CR, and DT on TTR is significant; (2) PR and CR have a positive effect on TTR, and the average improvement rate is about $77.03 \%$ and $73.20 \%$ with the increase of PR and CR, respectively; (3) meanwhile CL and DT have a negative effect on TTR, and TTR decreased by $31.21 \%$ as DT increased from 0 to $180 \mathrm{~s}$. 
There is a limitation for this paper, because only five characteristics indexes are analyzed. In future studies, more CV characteristics indexes should be selected to explore the impact of the characteristic indexes on the TTR of road networks. Simulation should still be extended, providing further comparison in detail.

\section{Conflicts of Interest}

The authors declare that there are no conflicts of interest regarding the publication of this paper.

\section{Acknowledgments}

This work was financially supported by the National Natural Science Foundation of China (Grant nos. 61473028, 71621001), Beijing Municipal Natural Science Foundation (Grant no. 8162031), and the National High Technology Research and Development Program of China ("863" Program) (Grant no. 2015AA124103).

\section{References}

[1] J. Ma, X. Li, F. Zhou, J. Hu, and B. B. Park, "Parsimonious shooting heuristic for trajectory design of connected automated traffic part II: computational issues and optimization," Transportation Research Part B: Methodological, vol. 95, pp. 421-441, 2017.

[2] F. Zhou, X. Li, and J. Ma, "Parsimonious shooting heuristic for trajectory design of connected automated traffic part I: theoretical analysis with generalized time geography," Transportation Research Part B: Methodological, vol. 95, pp. 394-420, 2017.

[3] L. Du, L. Han, and S. Chen, "Coordinated online in-vehicle routing balancing user optimality and system optimality through information perturbation," Transportation Research Part B: Methodological, vol. 79, pp. 121-133, 2015.

[4] Y. Asakura and M. Kashiwadani, "Road network reliability caused by daily fluctuation of traffic flow," in Proceedings of the 19th PTRC Summer Annual Meeting, University of Sussex, Brighton, UK, 1991.

[5] A. Tavassoli Hojati, L. Ferreira, S. Washington, P. Charles, and A. Shobeirinejad, "Modelling the impact of traffic incidents on travel time reliability," Transportation Research Part C: Emerging Technologies, vol. 65, pp. 49-60, 2016.

[6] J. Q. Leng, Travel Time Reliability of Urban Road Network under Ice and Snowfall Conditions, Harbin Institute of Technology, 2010.

[7] L. Wu, X.-H. Li, J. Wang, and Z.-B. Liu, "Reliability evaluation on travel time in freeway network under fog environment influence," Journal of PLA University of Science and Technology, vol. 11, no. 2, pp. 233-238, 2010.

[8] J. Wang, J. He, and L. Wu, "Evaluating approach of travel time reliability for highway network under rain environment," Journal of Transportation Systems Engineering and Information Technology, vol. 11, no. 6, pp. 117-123, 2011.

[9] J. Lee and B. Park, "Evaluation of route guidance strategies based on vehicle-infrastructure integration under incident conditions," Transportation Research Record, no. 2086, pp. 107-114, 2008.
[10] X. Ma, Y.-J. Wu, and Y. Wang, "DRIVE net: E-science transportation platform for data sharing, visualization, modeling, and analysis," Transportation Research Record, no. 2215, pp. 3749, 2011.

[11] X. L. Ma, Z. M. Tao, Y. H. Wang, H. Y. Yu, and Y. P. Wang, "Long short-term memory neural network for traffic speed prediction using remote microwave sensor data," Transportation Research Part C: Emerging Technologies, vol. 54, no. 1, pp. 187-197, 2015.

[12] S. Wang and X. Qu, "Station choice for Australian commuter rail lines: equilibrium and optimal fare design," European Journal of Operational Research, vol. 258, no. 1, pp. 144-154, 2017.

[13] Y. Iida, "Basic concepts and future directions of road network reliability analysis," Journal of Advanced Transportation, vol. 33, no. 2, pp. 125-134, 1999.

[14] H. X. Liu, L. Bu, and Y. Pu, "Travel time reliability on stochastic road network," China Civil Engineering Journal, vol. 37, no. 8, pp. 102-105, 2004.

[15] J. Y. T. Wang, M. Ehrgott, and A. Chen, "A bi-objective user equilibrium model of travel time reliability in a road network," Transportation Research Part B: Methodological, vol. 66, pp. 415, 2014.

[16] Y. L. Pei and C. Y. Gai, "Study on operation reliability of highway network," Journal of. Highway and Transportation Research and Development, vol. 22, no. 5, pp. 119-123, 2005.

[17] J. S. Chang, "Assessing travel time reliability in transport appraisal," Journal of Transport Geography, vol. 18, no. 3, pp. 419425, 2010.

[18] H. Tu, H. Li, H. van Lint, and H. van Zuylen, "Modeling travel time reliability of freeways using risk assessment techniques," Transportation Research Part A: Policy and Practice, vol. 46, no. 10, pp. 1528-1540, 2012.

[19] K. Chen, Models and Algorithms for Travel Time Reliability Assessment of Urban Road Networks Based-on Moving Source Data, Jiaotong University, Beijing, China, 2008.

[20] M. Bell, C. Cassir, Y. Iida et al., "A sensitivity based approach to network reliability assessment," in Proceedings of the 14th International Symposium on Transportation and Traffic Theory, 1999.

[21] X. Qu, S. Wang, and J. Zhang, "On the fundamental diagram for freeway traffic: a novel calibration approach for single-regime models," Transportation Research Part B: Methodological, vol. 73, pp. 91-102, 2015.

[22] X. Qu and S. Wang, "Long-distance-commuter (LDC) lane: a new concept for freeway traffic management," Computer-Aided Civil and Infrastructure Engineering, vol. 30, no. 10, pp. 815-823, 2015.

[23] X. Qu, Y. Yang, Z. Liu, S. Jin, and J. Weng, "Potential crash risks of expressway on-ramps and off-ramps: a case study in Beijing, China," Safety Science, vol. 70, pp. 58-62, 2014.

[24] Y. Asakura, "Reliability measures of an origin and destination pair in a deteriorated road network with variable flows: transportation networks: recent methodological advances," in Proceedings of the 4th Meeting of the EURO Working Group in Transportation, pp. 273-287, Pergamon Press, Helsinki, Finland, 1999.

[25] F. Yang, M. P. Yun, and X. G. Yang, "Single lane microscopic traffic flow model based on multi-agent in CVIS circumstance," Journal of Tongji University (Natural science), vol. 40, no. 8, pp. 1189-1196, 2012.

[26] H. Noori and M. Valkama, "Impact of VANET-based V2X communication using IEEE 802.11p on reducing vehicles traveling 
time in realistic large scale urban area," in Proceedings of the 2nd IEEE International Conference on Connected Vehicles and Expo (ICCVE '13), pp. 654-661, December 2013.

[27] S. Nie, Simulation on the Interaction Relationship between Information Flow and Traffic Flow in the Inter-Vehicle Communication Scenario, Beijing Jiaotong University, 2013.

[28] E. Paikari, S. Tahmasseby, and B. Far, "A simulation-based benefit analysis of deploying connected vehicles using dedicated short range communication," in Proceedings of the 25th IEEE Intelligent Vehicles Symposium (IV '14), pp. 980-985, Dearborn, Mich, USA, June 2014.

[29] M. W. Ng, W. Y. Szeto, and S. Travis Waller, "Distribution-free travel time reliability assessment with probability inequalities," Transportation Research Part B: Methodological, vol. 45, no. 6, pp. 852-866, 2011.

[30] M. Zhou, X. Qu, and S. Jin, "On the impact of cooperative autonomous vehicles in improving freeway merging: a modified intelligent driver model-based approach," IEEE Transactions on Intelligent Transportation Systems, vol. 99, pp. 1-7, 2016. 


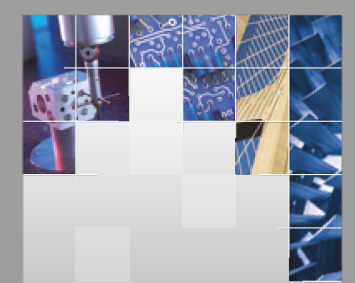

\section{Enfincering}
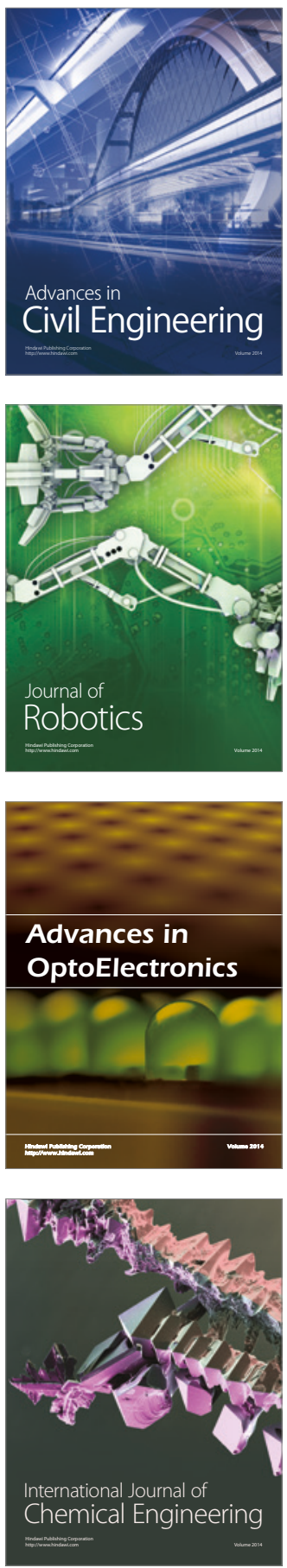

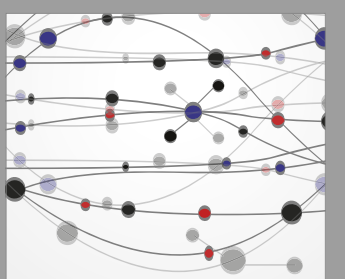

The Scientific World Journal

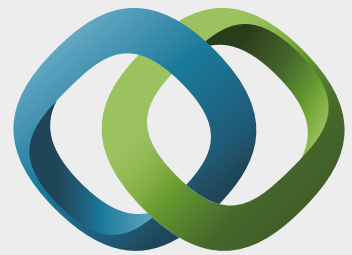

\section{Hindawi}

Submit your manuscripts at

https://www.hindawi.com
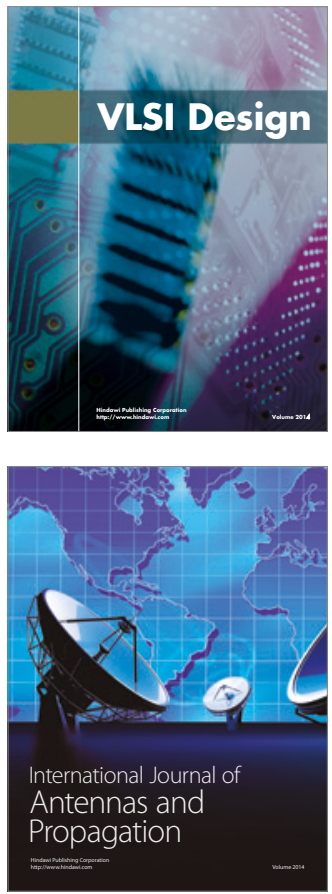

\section{Rotating}

Machinery
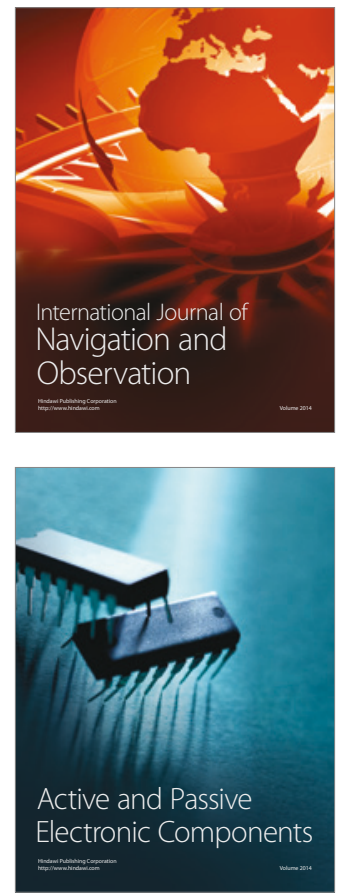
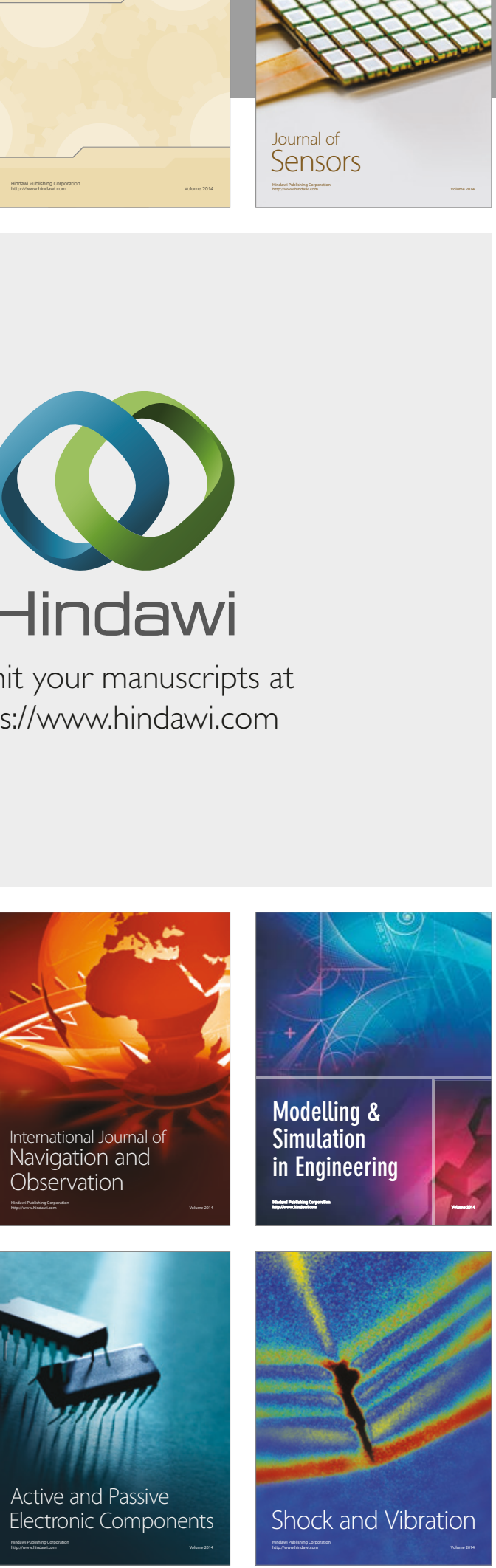
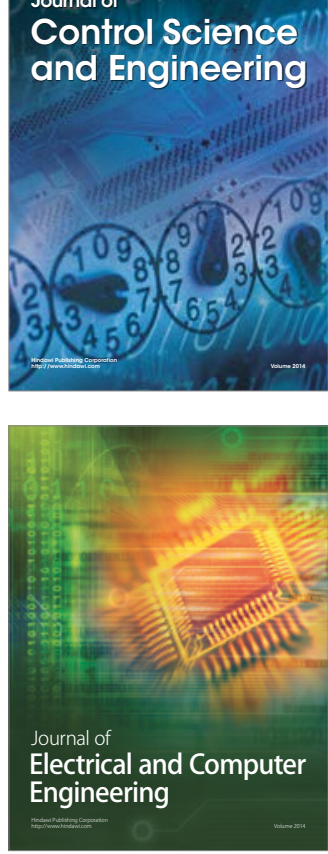

Distributed

Journal of

Control Science

and Engineering
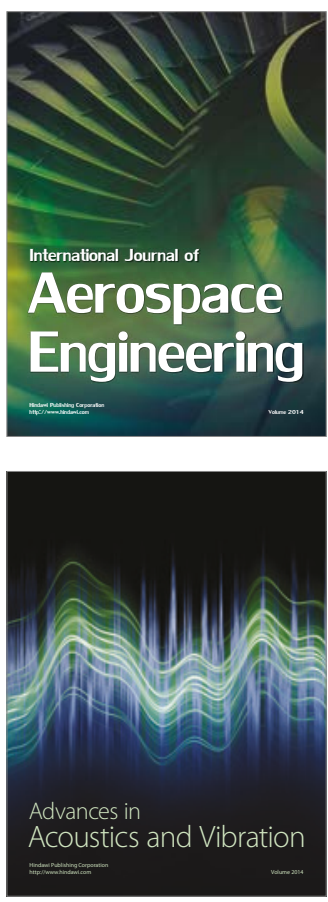

Sensor Networks 